\title{
Superconducting resonator used as a beam phase detector
}

\author{
S. I. Sharamentov, R. C. Pardo, P. N. Ostroumov, B. E. Clifft, and G. P. Zinkann \\ Argonne National Laboratory, 9700 S. Cass Avenue, Argonne, Illinois 60439, USA
}

(Received 14 April 2003; published 9 May 2003)

\begin{abstract}
Beam-bunch arrival time has been measured for the first time by operating superconducting cavities, normally part of the linac accelerator array, in a bunch-detecting mode. The very high $Q$ of the superconducting cavities provides high sensitivity and allows for phase-detecting low-current beams. In detecting mode, the resonator is operated at a very low field level comparable to the field induced by the bunched beam. Because of this, the rf field in the cavity is a superposition of a "pure" (or reference) rf and the beam-induced signal. A new method of circular phase rotation (CPR), allowing extraction of the beam phase information from the composite rf field was developed. Arrival time phase determination with CPR is better than $1^{\circ}$ (at $48 \mathrm{MHz}$ ) for a beam current of $100 \mathrm{nA}$. The electronics design is described and experimental data are presented.
\end{abstract}

DOI: 10.1103/PhysRevSTAB.6.052802

\section{INTRODUCTION}

Resonant rf cavities are widely used in the accelerator community $[1,2]$ for beam detection. The simplest applications extract only beam intensity from the amplitude of the induced rf signal [3]. More sophisticated applications rely on accurately determining the phase of the induced rf signal to measure the beam arrival time at the detection cavity or, effectively, the time of flight (TOF) between two cavities. If the amplitude of the induced rf field is compared in resonant cavities operating at two or more significantly different harmonics, then bunch width information may also be obtained [4]. Up to now, such systems have been based on a variety of roomtemperature normal-conducting cavities.

Superconducting (SC) linear accelerators have become widely popular for the acceleration of all types of charged particles [5-8] due to their high accelerating gradients and low operating costs. The design of these linacs relies on the use of short independently phased cavities with transverse focusing elements interspersed among the resonators. For optimum beam optics, close spacing of the active elements is needed with minimum drift distances. This requirement is especially true in the low-velocity $(\beta \leq 0.1)$ accelerator region for heavy ions with low charge-to-mass ratio $(q / \mathrm{m})$, and so the pressure to minimize the space needed for diagnostics is intense.

In existing heavy-ion superconducting linacs designed for maximum transverse and longitudinal acceptance such as ATLAS, no space has been allocated for diagnostics over $10 \mathrm{~m}$ distances. At low beam velocities, beam steering, focusing effects, and long drift spaces make longitudinal diagnostics cumbersome and can induce distortions in the data unrelated to the actual beam conditions in the early resonators. This problem will be even more acute in SC linacs planned for future large facilities such as the Rare Isotope Accelerator [9].

In this paper, we demonstrate for the first time the use of a superconducting resonator, normally used for beam
PACS numbers: 83.10.Pp, 85.25.-j, 41.75.-i, 41.85.Qg

acceleration, to detect the arrival time of a beam bunch. This is accomplished when the resonator is operating, not as an accelerating cavity, but at a very low field level, comparable to the field induced by the beam pulse traversing the cavity. In this bunch-detection mode, the timing information obtained from the detecting resonator can be used to accurately determine the beam-rf phase relationship in an upstream resonator and in this way use the elements of the linac itself to provide a complete and conveniently distributed set of beam phase detectors for accurately and quickly tuning the linac.

\section{SUPERCONDUCTING RESONATOR FEATURES}

Superconducting resonators (SCR) are resonant zstructures with a very high quality factor, implying a very low frequency bandwidth. Their intrinsic $Q$ can reach $10^{9}-10^{10}$, depending on the eigenfrequency and type of resonator. Even loaded frequency bandwidths typically lie within a $0.1-10 \mathrm{~Hz}$ range, for different eigenfrequencies.

A fundamental control problem with SCRs is microphonic fluctuations of the resonator's resonant frequency caused by the mechanical vibration of superconducting system components. Mechanical vibration in the system can be viewed as random mechanical noise with a Gaussian spectral distribution [10]. The rms value of the microphonics spectrum width typically is comparable to or even much greater than the SCR frequency bandwidth.

These effects prevent direct use of the induced rf signal in the SCR for beam phase detection. Similar to the normal SCR application for particle acceleration, beam phase detection requires stabilization of the SCR resonant frequency, which is typically accomplished by means of $\mathrm{rf}$ feedback loops. Another way of minimizing microphonics is to decrease the $Q$ value of the SCR. This can be done, for example, by overcoupling an SCR drive port. For the SCR application as a beam phase detector, 
this method cannot be recommended, however, since it results in lower sensitivity.

To achieve normal operation of the feedback loops, some initial level of rf field inside the resonator is required. The level of the rf field has to be as low as possible. Otherwise the energy of the incoming beam can be changed due to the interaction between the beam and cavity's rf field.

An important realization follows from this argument. Unlike "normal" beam phase detectors, the rf field in an SCR is always a superposition of a pure (or reference) $\mathrm{rf}$ and the beam-induced signal.

Summarizing, using an SCR as a beam phase pickup, the following three conditions are required:

(i) Both amplitude and phase feedback loops have to be locked-some rf is required.

(ii) Reference rf level has to be low.

(iii) Resonator rf field is a superposition of a reference rf field and beam-induced field.

\section{BEAM PHASE MEASUREMENTS WITH SCR}

A graphical interpretation of the superposition of a beam-induced signal and a reference rf field is shown in Fig. 1. The magnitude and phase of the beam-induced signal is denoted as $A_{b}, \phi_{b}$, and the magnitude and phase of the reference rf field as $A_{\mathrm{rf}}, \phi_{\mathrm{rf}}$. The resultant resonator rf vector can be written as

$$
A_{\Sigma}(t) \exp \left[i \phi_{\Sigma}(t)\right]=A_{\mathrm{rf}} \exp \left[i \phi_{\mathrm{rf}}(t)\right]+A_{b} \exp \left(i \phi_{b}\right)
$$

with amplitude and phase

$$
\begin{aligned}
A_{\Sigma}(t) & =A_{\mathrm{rf}} \sqrt{1+p^{2}+2 p \cos \left(\phi_{\mathrm{rf}}-\phi_{b}\right)}, \\
\phi_{\Sigma}(t) & =\arctan \frac{\sin \phi_{\mathrm{rf}}+p \sin \phi_{b}}{\cos \phi_{\mathrm{rf}}+p \cos \phi_{b}},
\end{aligned}
$$

where $p=A_{b} / A_{\mathrm{rf}}$ is the relative beam-induced signal amplitude. We will consider the case $p<1$ only, because practically for any beam current, the amplitude of the reference rf field can be chosen to satisfy this condition.

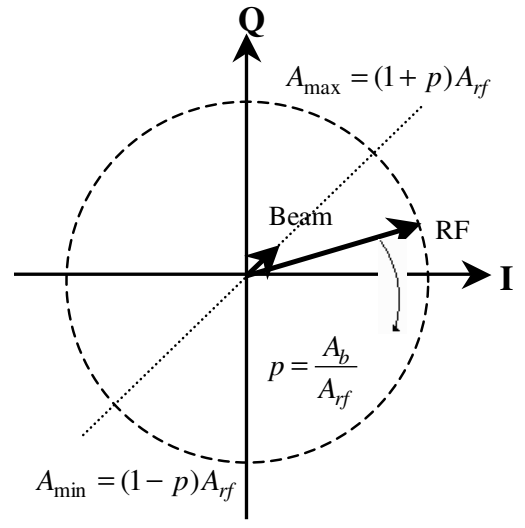

FIG. 1. Graphical illustration of the superposition of the beam-induced signal and reference rf.
For small $p$, the amplitude of the resultant field is

$$
A_{\Sigma}(t) \approx A_{\mathrm{rf}}\left[1+p \cos \left(\phi_{\mathrm{rf}}-\phi_{b}\right)\right] .
$$

As follows from Eqs. (2), neither amplitude nor phase of the resonator rf field can give unambiguous information about the beam phase, because both amplitude and phase of the resultant $\mathrm{rf}$ field are functions of the reference rf field amplitude $A_{\mathrm{rf}}$ and phase $\phi_{\mathrm{rf}}$.

Nevertheless, there is a simple way to extract beam phase information from the resultant $\mathrm{rf}$ field. Suppose we apply a linear circular phase modulation to the reference rf field:

$$
\phi_{\mathrm{rf}}(t)=\omega_{r} t=2 \pi F_{r} t
$$

which can be illustrated in Fig. 1 as a continuous circular rotation of the $\mathrm{rf}$ vector with modulation frequency $F_{r}$. Then, the magnitude of the resultant rf field will be amplitude modulated with the same frequency $F_{r}$, and, relative to the reference signal $\cos \omega_{r} t$, will be shifted in phase by an angle exactly equal to the beam phase $\phi_{b}$ :

$$
A_{\Sigma}(t)=A_{\mathrm{rf}}\left[1+p \cos \left(\omega_{r} t-\phi_{b}\right)\right], \quad p<1 .
$$

Thus, by applying a linear circular phase rotation to the reference rf field, and measuring the phase shift between the resonator's resultant signal magnitude and modulation signal, one can measure the beam-induced signal phase. This conclusion is also valid for larger $p$, as shown in Fig. 2. For higher values of $p$ close to 1 , the amplitude modulation is no longer a harmonic waveform, but the phase of the first harmonic of the modulated waveform is still shifted by a beam phase angle $\phi_{b}$.
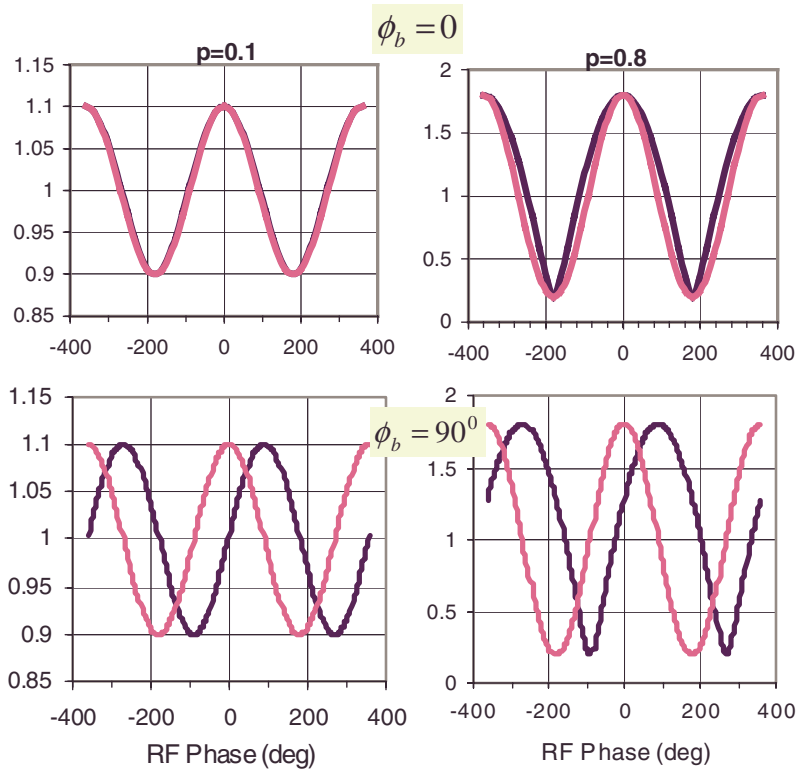

FIG. 2. (Color) Envelope of resonator resultant field for different beam amplitude and phase, relative units. $F_{r}=1 \mathrm{~Hz}$. Red curve: reference signal. 
It is interesting to notice that this proposed method of circular phase modulation is somewhat similar to a wellknown method of frequency conversion, widely used in the TOF technique, for measuring longitudinal beam parameters, such as energy, time of arrival, etc. For instance, the ATLAS superconducting accelerator has a TOF system for measuring beam energy, which is based on using special warm resonators as beam phase pickups, and subsequent countdown frequency conversion by a value of 3-10 kHz, depending on the resonator's rf frequency [11]. Circular phase modulation is equivalent to frequency conversion with an intermediate frequency $F_{r}$, because circular phase rotation with rate $F_{r}$ shifts the rf carrier frequency by $F_{r}$. The value of $F_{r}$ used in this application is $5-10 \mathrm{~Hz}$, because of the limited bandwidth of the SCR feedback loops.

\section{ATLAS BEAM PHASE-DETECTION SETUP}

All beam phase-detection tests were made at the first Positive Ion Injector (PII) cryostat, which has six superconducting quarter-wave resonators with central frequency $48.5 \mathrm{MHz}$. Their position numbers are $R 111$, $R 112, \ldots, R 116$. Figure 3 shows a simplified rf diagram of an ATLAS superconducting resonator in normal accelerating mode. The resonator operates in a self-excited mode, with locked amplitude and phase feedback loops. Phase locking is accomplished by means of a voltagecontrolled reactance VCX [12]. For low field operation [condition (ii) from Sec. II], an additional $40 \mathrm{~dB}$ gain preamplifier and a $20 \mathrm{~dB}$ attenuator were installed in the resonator pickup line and the power amplifier input line, as shown in Fig. 4. The preamplifier and attenuator allow normal operation of the rf control module and rf power amplifier, while the resonator field is only $1 \%$ of the normal accelerating mode. This background field can

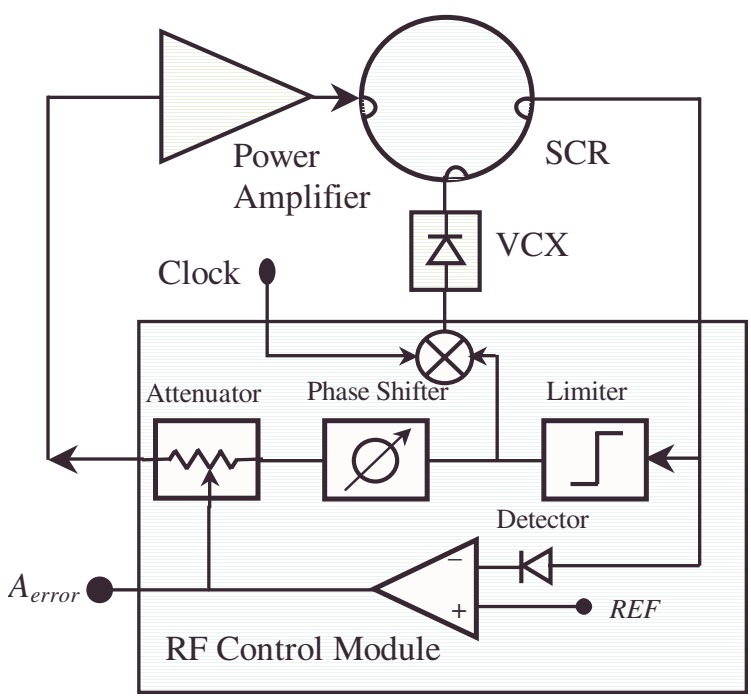

FIG. 3. (Color) Simplified rf diagram of the ATLAS superconducting resonator in accelerating mode.

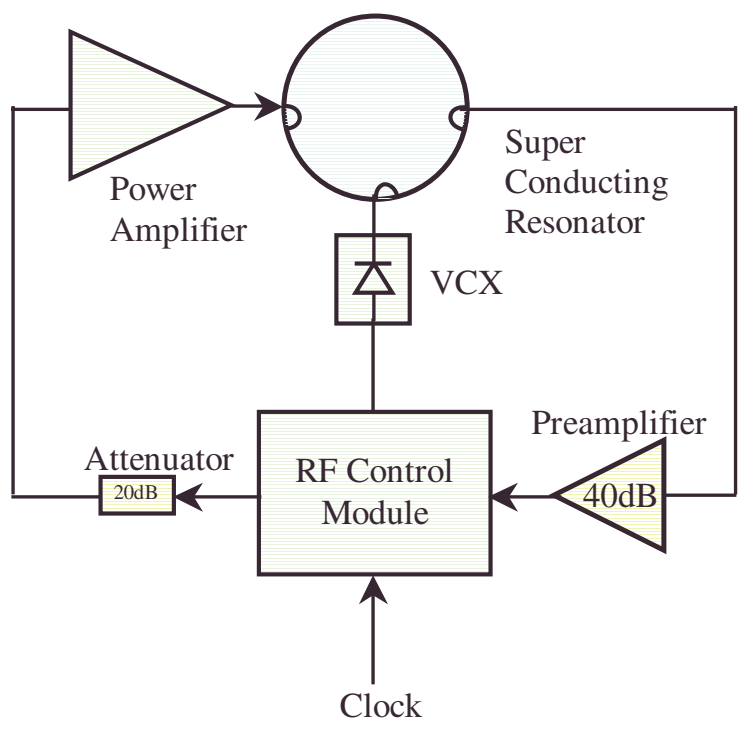

FIG. 4. (Color) SCR low field operation simplified diagram.

cause acceleration of the beam in the detecting resonator and affect the effective arrival time in the resonator slightly. For the worst case situation, where the velocity is still very low $(\beta \sim 0.015 c)$ and the prior total voltage is low, the observed phase can be perturbed by as much as $2^{\circ}$. For all other cases, this perturbation is less than $0.5^{\circ}$. This type of perturbation in interpreting the measured phase can be corrected by calculating the expected effect, but was not included in these early studies.

The complete experimental setup for using a SCR as a beam phase detector is shown in Fig. 5. An external

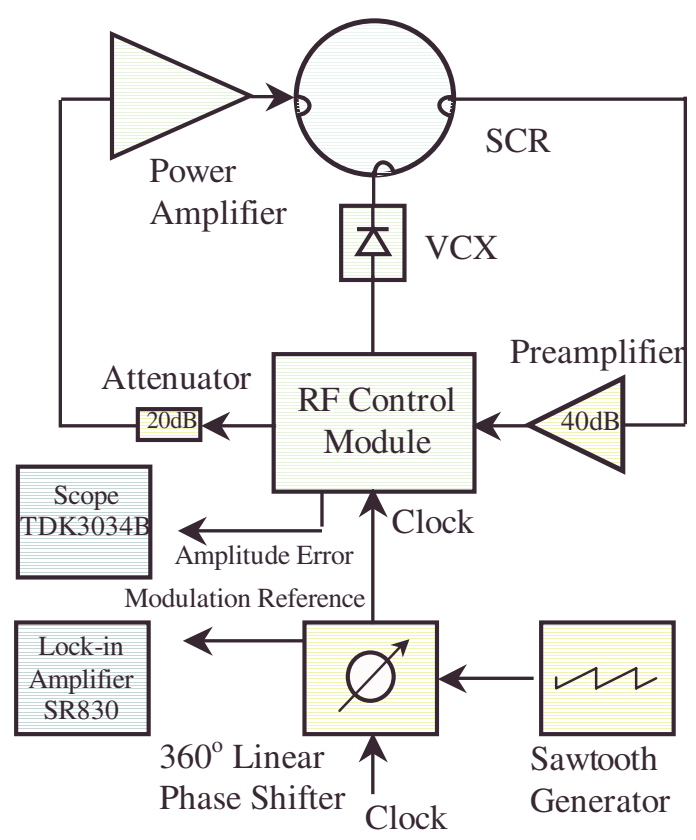

FIG. 5. (Color) Complete experimental setup for using SCR as a beam phase detector. 


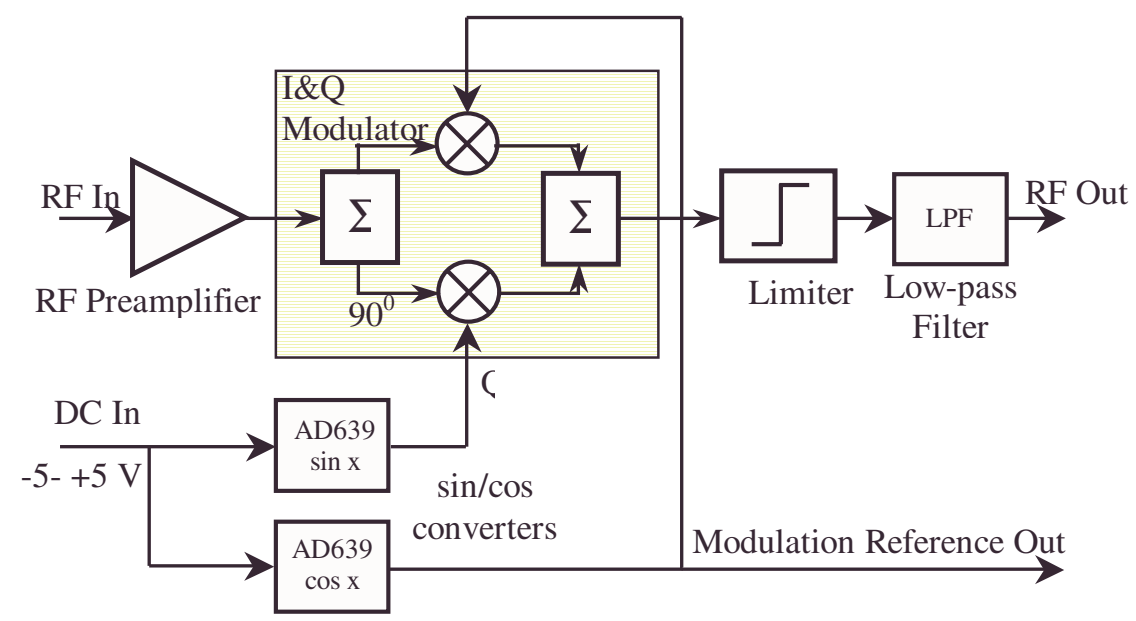

FIG. 6. (Color) Block diagram of a $360^{\circ}$ linear phase shifter. Minicircuits MIQC-60WD I\&Q demodulator and analog devices AD639 trigonometric converters provide high linearity of the phase control curve.

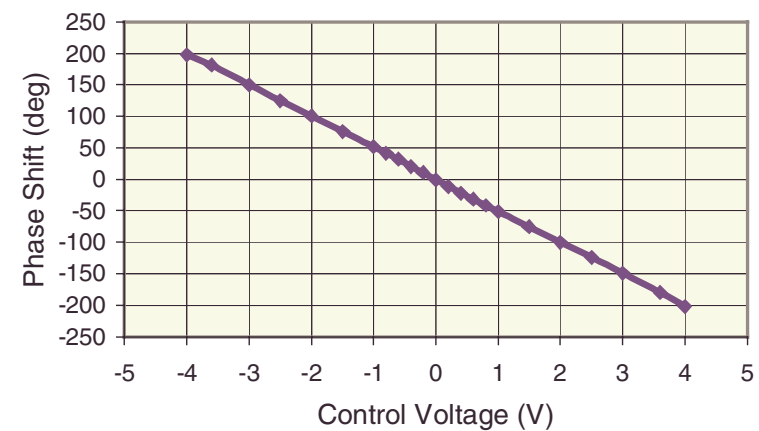

FIG. 7. (Color) Phase shifter's control curve. Phase slope is $50^{\circ} / \mathrm{V}$.

linear phase shifter with $360^{\circ}$ range controlled by a sawtooth shape signal was installed in the master oscillator (clock) line. The detailed structure and control curve of the phase shifter are shown in Figs. 6 and 7. Parameters of the phase modulation were adjusted in such a way as to get continuous circular rotation of the reference $\mathrm{rf}$ field vector in the resonator, with a $6-7 \mathrm{~Hz}$ rate. Because of the interaction between the rotating reference $\mathrm{rf}$ field vector and the beam-induced rf vector inside the resonator, an amplitude error signal in the amplitude feedback loop was also modulated. It was found that the amplitude error signal is very convenient to use as the beam phase signal, because it reflects any changes in the resonator's rf field magnitude, and can be amplified to any desired level. Examples of amplitude error modulation are shown in Fig. 8.

A synchronous detection method was chosen for extracting the beam phase information, because it provides high accuracy phase measurements in a noisy environment, which is typically the case for SCR due to the high level of microphonics. A Stanford Research Lab SR830 two-channel lock-in amplifier was used as the

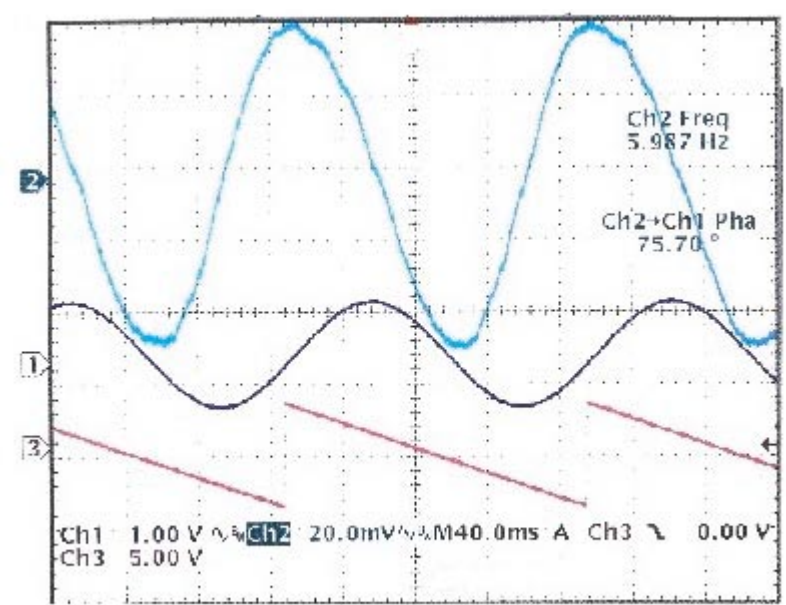

(a)

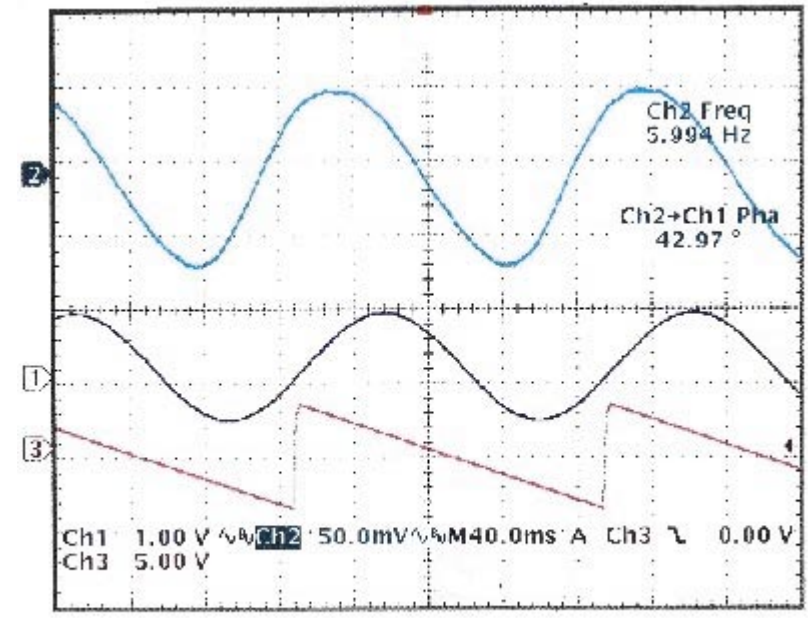

(b)

FIG. 8. (Color) Examples of resonator's resultant field modulation, (a) $R 111$ tune, monitor at $R 112$ position; (b) $R 114$ tune, monitor at $R 116$ position. Top traces: amplitude feedback loop error signal; middle traces: modulation reference signal; bottom traces: sawtooth control voltage. Time scale is $40 \mathrm{~ms} / \mathrm{div}$. 
synchronous detector, and a Tektronix digital TDK3034B scope was chosen for waveform display. Overall accuracy of the beam phase measurements in the real ATLAS accelerator noise environment is estimated to be better than half a degree.

\section{EXPERIMENTAL RESULTS}

The most difficult region of the ATLAS linac to tune with our present surface barrier detector diagnostics is the lowest energy section of the PII linac. This is due to steering effects from misalignments as well as asymmetric fields inherent in the resonators and the relatively long distance $(\sim 10 \mathrm{~m})$ to the diagnostics region at the end of the PII. Therefore, we chose to focus on this very lowvelocity region of the linac to develop and test this new system. Figure 9 shows the PII and includes a blowup of the first six resonators in the first cryostat of PII used in these measurements.

First beam phase measurements were made using a ${ }^{20} \mathrm{Ne}^{8+}$ beam in March 2002. Electrical beam current was about $1 \mu \mathrm{A}$. The beam was delivered from the ATLAS Electron Cyclotron Resonance ion source injector with a velocity of $0.0085 c$ and bunched to approximately $0.25 \mathrm{~ns}$ FWHM by a two-stage bunching system [13]. The idea was to operate a nearby downstream SCR as the beam phase monitor, as described before, and observe the induced signal phase as a function of the rf phase of an upstream resonator. Figure 10 shows experimental data for the cases where resonators $R 112, R 113$, and $R 114$ were alternately used as the beam phase monitor, and the first resonator, $R 111$, was turned on with $\mathrm{rf}$ field at approximately $3 \mathrm{MV} / \mathrm{m}$. The fact that for all three locations of the beam phase monitor, the measured phase curves have a similar shape, and their minimum

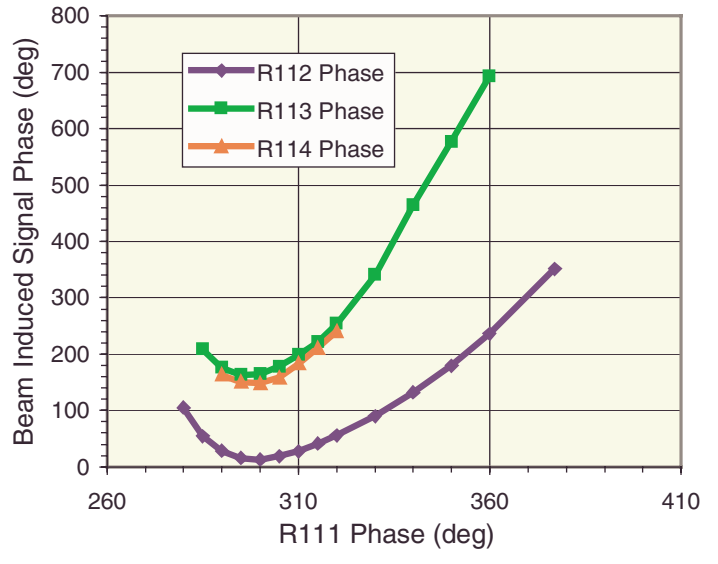

a

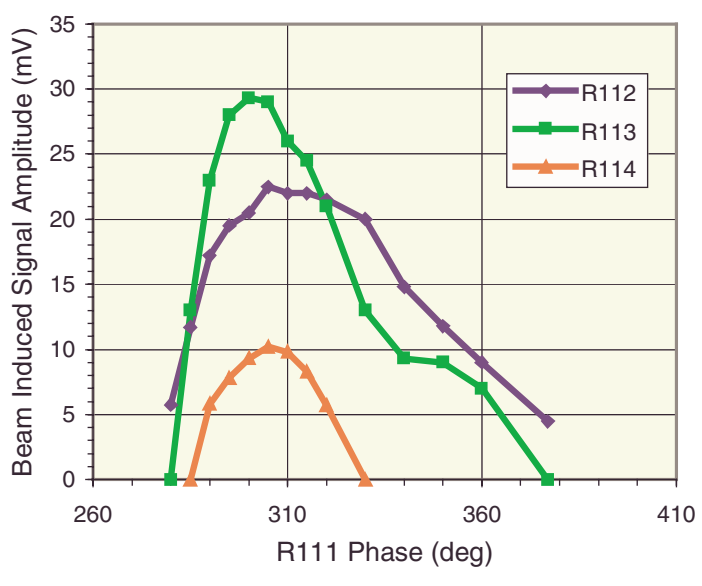

b

FIG. 10. (Color) Beam-induced signal phase (a) and magnitude of amplitude error modulation (b) as a function of the $R 111 \mathrm{rf}$ phase. Beam phase monitor was placed at three different locations, $R 112, R 113$, and $R 114$.

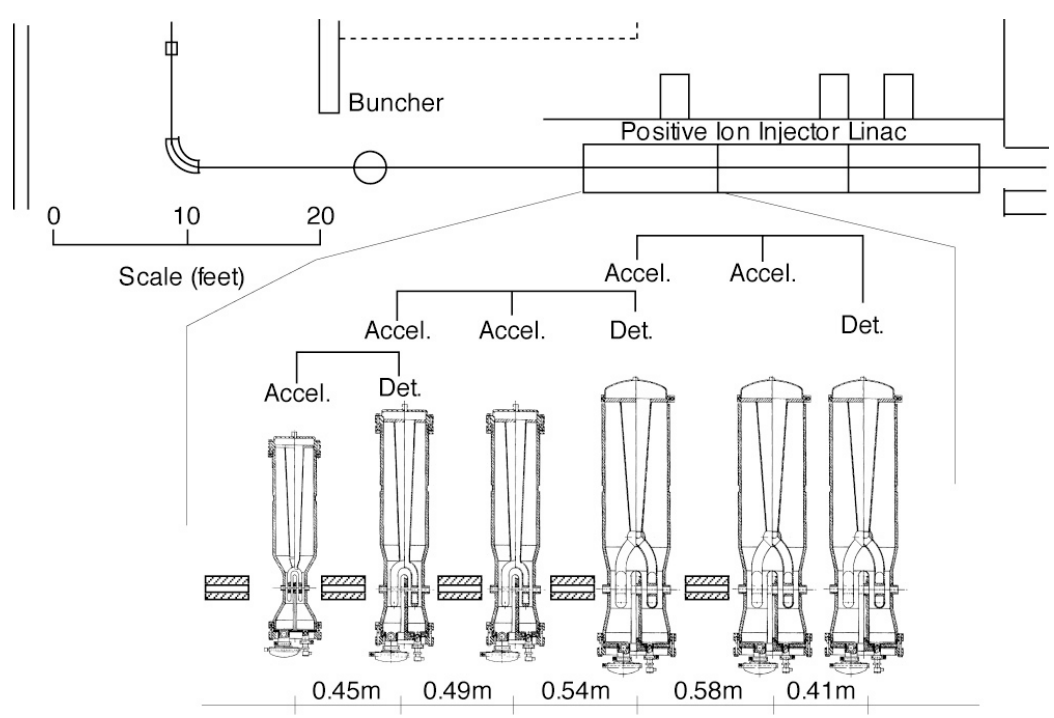

FIG. 9. Floor plan of the ATLAS Positive Ion Injector and a blowup of the resonator configuration in the first cryostat used to develop the techniques described in this paper. 
TABLE I. Comparison of phase-shift energy determination and solid-state detector measured energy.

\begin{tabular}{ccccc}
\hline \hline $\begin{array}{c}\text { Resonator pair } \\
\text { (accelerating/detecting) }\end{array}$ & $\begin{array}{c}\text { Distance } \\
(\mathrm{m})\end{array}$ & $\begin{array}{c}\text { Total phase shift } \\
(\mathrm{deg}) /(\mathrm{ns})\end{array}$ & $\begin{array}{c}\text { Calculated energy } \\
(\mathrm{MeV})\end{array}$ & $\begin{array}{c}\text { Measured energy } \\
(\mathrm{MeV})\end{array}$ \\
\hline$R 113 / R 114$ & 0.540 & $221 / 12.7$ & 7.2 & 7.7 \\
$R 114 / R 116$ & 0.991 & $616 / 35.3$ & 12.6 & 12.7 \\
$R 115 / R 116$ & 0.406 & $104 / 6.0$ & 16.2 & 16.5 \\
\hline \hline
\end{tabular}

corresponds to the same value of the $R 111$ phase of $300^{\circ}$, is a confirmation that the measured data were real beam phase data. Another important conclusion from the beam phase data in Fig. 10 is that the value of $R 111 \mathrm{rf}$ phase $300^{\circ}$ corresponds to the point of maximum energy gain where the beam and resonator rf relative phase is $0^{\circ}$.

A semimanual version of this system was then used to "tune" the first five resonators in the PII linac by sequentially moving the electronics from one resonator to another in order to approximately maintain the velocity match condition and maintain a well bunched beam into the detecting resonator. A wide range of tunes was developed to match beams with charge-to-mass ratio $(q / \mathrm{A})$ from 0.15 to 0.375 . In each instance the actual energy gain was determined using a solid-state detector to measure the absolute energy gain from each resonator. The combination of the accelerating resonator to be tuned and the detecting resonator used is shown in Fig. 9 along with the relevant dimensions.

In principle, the total phase shift observed at the detecting resonator between the last accelerating resonator in "on" and "off" states is the beam transit time change due to the energy gain given to the beam by that resonator. The energy gain of the beam is calculable within that model and the total beam energy at that point can be determined. The measured total phase shift and the calculated energy of the beam after acceleration are presented in Table I for a ${ }^{40} \mathrm{Ar}^{6+}$ beam. Table I shows data identified by the last accelerating resonator and the detecting resonator. The energy of the ${ }^{40} \mathrm{Ar}$ beam into the first of this set of resonators was $5.5 \mathrm{MeV}$.

If two resonators are used in the detection mode, then the difference in phase contains time-of-flight information, which can be used to obtain the absolute energy directly. A calibration procedure would first need development in order to determine the electronic phase offset between the two detecting resonators. Measuring only the phase shift eliminates the need for that calibration, but errors now propagate through the data reducing the precision of energy determinations with the technique presented here.

\section{CONCLUSION}

The system described in this paper is now in regular use at the ATLAS facility for tuning the resonators in the lowest energy section of the facility. The system is a significant improvement over the previous diagnostics system located 8-10 $\mathrm{m}$ downstream from these early resonators. Full integration of the method into the ATLAS control system is now underway. Improved phase sensitivity at higher energy may be possible where the technique can be used at the zero-crossing, $90^{\circ}$, point where the maximum possible phase sensitivity exists. At the low velocities studied in this report, the debunching in that phase region makes the detecting resonator insensitive to the beam bunches.

\section{ACKNOWLEDGMENTS}

This work was supported by the U.S. Department of Energy under Contract No. W-31-109-ENG-38.

[1] R. N. Lewis, Nucl. Instrum. Methods 151, 371 (1978).

[2] J.S. Kim, C. D. Nantista, D. H. Whittum, R. H. Miller, S. G. Tantawi, and A.W. Weidemann, in Proceedings of the Particle Accelerator Conference, Chicago, IL, 2001, edited by Peter Lucas (IEEE, Piscataway, NJ, 2001), p. 1345.

[3] R. Lorenz, in Proceedings of the IEEE Particle Accelerator Conference, Washington, D.C., 1993, edited by S.T. Corneliussen (IEEE, Piscataway, NJ, 1993), p. 2325 .

[4] R. Pardo, B. E. Clifft, P. DenHartog, D. Kovar, W. Kutschera, and K. E. Rehm, Nucl. Instrum. Methods Phys. Res., Sect. A 270, 226 (1988).

[5] L. M. Bollinger et al., Nucl. Instrum. Methods Phys. Res., Sect. B 79, 753 (1993).

[6] G. A. Kraft, in Proceedings of the 1994 International Linac Conference, Tsukuba, Japan (unpublished), p. 9.

[7] R. L. Custom, in Proceedings of the XX International Linac Conference, Monterey, CA, 2000, edited by A.W. Chao (Stanford Linear Accelerator Center, Stanford, CA, 2001), p. 321.

[8] K.W. Shepard and T. E. Tretyakova, in Proceedings of the XX International Linac Conference, Monterey, CA, 2000 (Ref. [7]), p. 920.

[9] P. N. Ostroumov, J. A. Nolen, and K.W. Shepard, in Proceedings of the XX International Linac Conference, Monterey, CA, 2000 (Ref. [7]), p. 1018.

[10] G. Davis, J. Delayen, M. Drury, T. Hiatt, C. Hovater, T. Powers, and J. Preble, in Proceedings of the Particle 
Accelerator Conference, Chicago IL, 2001 (Ref. [2]), p. 1152.

[11] R. C. Pardo, R. N. Lewis, K.W. Johnson, and B. Clifft, IEEE Trans. Nucl. Sci. 30, 2237 (1983).

[12] J. M. Bogaty, B. E. Clifft, K.W. Sheppard, and G. P. Zinkann, in Proceedings of the IEEE Particle Accelerator Conference, Chicago, IL, 1989, edited by
F. Bennett and J. Kopta (IEEE, Piscataway, NJ, 1989), p. 1978 .

[13] P. K. Den Hartog, J. M. Bogaty, L. M. Bollinger, B. E. Clifft, R. C. Pardo, and K.W. Sheppard, in Proceedings of the IEEE Particle Accelerator Conference, Chicago, IL, 1989 (Ref. [12]), p. 545. 